\title{
A new training simulator for improved voltage control of the Hydro-Québec system
}

\author{
T. Van Cutsem, senior member, IEEE, G. Hassé, C. Moors, \\ S. Guillon, member, IEEE and R. Mailhot
}

\begin{abstract}
This paper reports on the development of a training simulator focusing on voltage control and stability. It has been derived from a quasi steady-state simulation software already used and validated in planning and operational planning studies. The computational engine has been provided with a user interface built on the client-server architecture, allowing the simulator to run on a network of PCs in a very flexible way. New displays can be set up easily and quickly. This tool is presently used by Hydro-Québec to improve the operator's ability to control transmission voltages. Other features, uses and benefits are reported.
\end{abstract}

Index Terms - voltage control, voltage stability, training simulator, long-tem dynamics, quasi steady-state simulation

\section{INTRODUCTION}

$\mathrm{T}$ he past and recent blackouts experienced throughout the world have emphasized the necessity to train operators to control power systems in normal and stressed conditions. Although their usage in control centers is not as generalized as it should be, Training Simulators (TS) are useful tools to improve the operators' skills. The latter can be trained to face situations that they (luckily) seldom meet in their everyday job. A sample of TS implementations is described in [1-8].

Although the available computer technology makes it possible to simulate in real time the whole dynamics of a power system [7,8], most TS rely on a model of the power long-term dynamics [1-6], which is sufficient for a wide range of applications, while less demanding in terms of data maintenance. To the authors' knowledge, most TS focus on the long-term dynamics of frequency (including system splitting and underfrequency load shedding). Power flow (PV/PQ bus) models of generators and loads are often used. While voltage control and stability is a critical aspect of power system operation, relatively few TS applications have been reported in this area.

Thierry Van Cutsem is with the Belgian National Fund for Scientific Research (FNRS) and the Department of Electrical Engineering and Computer Science (Montefiore Institute) of the University of Liège, Sart Tilman B37, B4000 Liège, Belgium (t.vancutsem@ulg.ac.be). As of working on this project, Gaëtan Hassé and Cédric Moors were with the same department.

Sébastien Guillon is with the Operational Planning department of the TransEnergie division of Hydro-Québec (guillon.sebastien@hydro.qc.ca). Richard Mailhot is with the Hydro-Québec control center, Complexe Desjardins, Tour de l'Est, CP 10,000 Montréal (Qc), Canada H5B 1H7 (mailhot.richard@hydro.qc.ca)
Training Simulators may take on the form of stand-alone applications $[1,2,7,8]$ or may be components of Energy Management Systems (EMS) [3-6]. In most cases, the TS displays are similar to those of the EMS, so that the user is placed in a control center replica. However, the TS that goes with the EMS may not meet the modeling needs of the power system under concern.

This paper reports on the development of SFR-ASTRE, a simple, stand-alone TS focusing (at least initially) on voltage control and long-term voltage stability. This simulator has been built from the ASTRE software, developed by the University of Liège and used for several years by HydroQuébec (H-Q) engineers in planning and operational planning voltage stability studies [9]. It relies on the Quasi SteadyState (QSS) approximation of the long-term dynamics [10], in which the short-term dynamics are replaced by equilibrium equations. Being much faster than real-time, this simulation has been modified to render the normal or an accelerated system evolution.

Next, the so modified computational engine has been provided with modules operating according to a client-server architecture. A server communicates with one or several instances of a graphic user interface running on a set of PCs connected through a local area network. Simply stated, each graphic interface consists of a background picture provided with control points and output displays. New displays can be easily and quickly developed, which may range from simple tables to detailed one-line diagrams. High flexibility was achieved with low software development efforts.

Starting from a software already used in planning and operational planning studies offered important advantages. Among them, let us quote : the direct access to well validated models and data, the very low additional data maintenance requirements, the possibility to rapidly construct a new training case from historical data files produced by the control center state estimator, and the readily available representation of the system protection schemes present on the H-Q system.

This simulator is now used at H-Q to improve the operators' capability to control transmission voltages. This is a challenging task owing to the capacitive behaviour of the long 735-kV lines, the high fluctuations of demand with time and the voltage stability limited operation of the system in its Southern part. Therefore, increasing the dispatchers' abilities to control voltages is an important goal for the technical support team. Good practices contribute to maximizing 
system security in normal and abnormal situations, minimizing the number of switching operations and decreasing the total active power losses.

Made available in 2003, this simulator was already used in the preparation of the 2003-2004 winter peak demand, where it has shown several of its expected benefits.

This paper is organized as follows. Section II explains the motivation for a TS at Hydro-Québec, while Section III describes its integration within the real-time and studies environments. Section IV describes the main features of the tool. Section V comments on a typical training session. Conclusion and perspectives are offered in Section VI.

\section{VoltAGE CONTROL OF THE HYDRO-QUEBEC SYSTEM}

\section{A. Transmission system}

The H-Q transmission system is characterized by long distances between the hydro-electric power generation and the load areas, including interconnection lines (see Fig. 1). A large amount of this power is transferred over $735-\mathrm{kV}$ seriescompensated transmission lines [11].

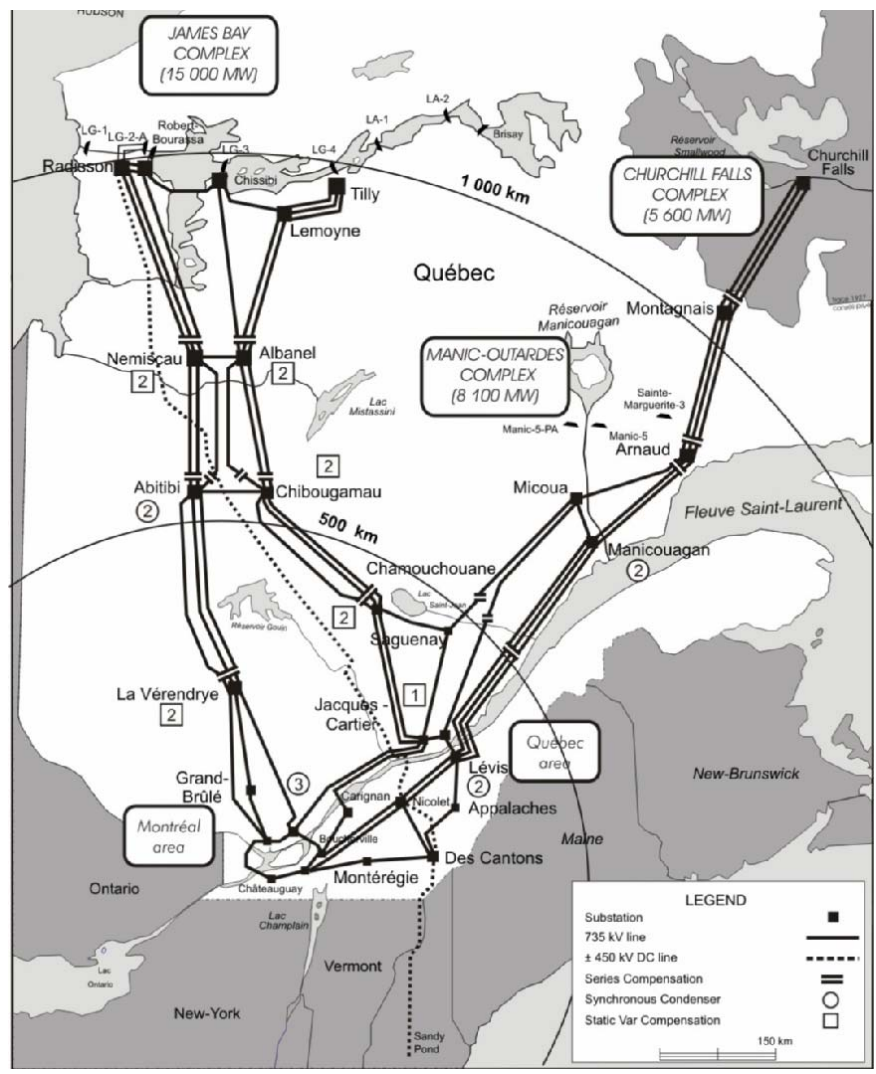

Fig. 1. Hydro-Quebec 735-kV transmission system

This particular configuration has developed over the years, first along a northeast axis, toward Manicouagan and Churchill Falls generating facilities (12000 MW), and then along a northwest axis, toward the James Bay power stations (15000 MW). The peak load is approximately $36000 \mathrm{MW}$.

\section{B. Voltage control}

The reliable and efficient control of $\mathrm{H}-\mathrm{Q}$ transmission voltages is demanding due to the capacitive nature of the long $735-\mathrm{kV}$ lines and the important variation of the demand.

The regulation is achieved by control center operators using static and dynamic shunt compensation, namely numerous reactors and capacitors, and 20 synchronous condensers and Static Var Compensators spread over the system (see Fig. 1).

The dynamic compensators are only partly dedicated to steady-state voltage regulation, most of their capacitive reserves being preserved for angle and long-term stabilization purposes. As a result, the dispatcher must maintain the voltage profile within the relatively narrow [730 750] $\mathrm{kV}$ range, while monitoring the reactive contributions of compensators.

A typical evolution during a high-demand day is shown in Fig. 2, obtained from real measurements taken on February 18,2004 . As shown by the figure, a load pick-up of around $3,000 \mathrm{MW}$ takes place in one hour, from about $6 \mathrm{~h} 30$ to $7 \mathrm{~h} 30$. Some days, the highest hourly variation can reach 5,000 MW.

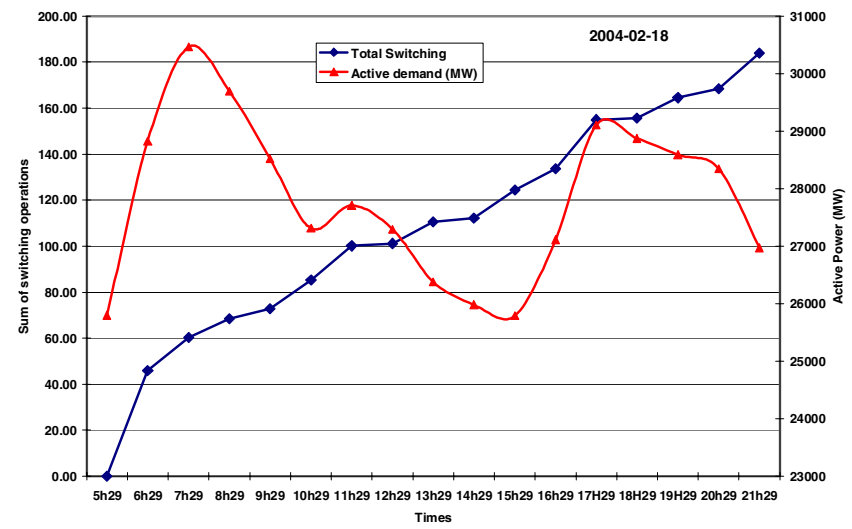

Fig.2. Evolution of active demand and cumulated number of switching operations over a high-demand day

Figure 2 also shows that the daily load variation required as many as 184 switching operations (involving 330-Mvar shunt reactors and 100-Mvar shunt capacitors), among which $30 \%$ were performed within one hour.

During that particularly high fluctuation period the system operator must react quickly [12], while taking into account the delay between its order and the effective switching.

In particular conditions, such as peak load period, system recovery following severe contingencies and/or operation of system protection schemes, the voltage control capability of system operators is even more crucial.

The above considerations illustrate the potential benefit of a TS to educate and support the HQ system operators even in normal operating conditions.

\section{Training objectives}

The TS described in this paper is intended to play a role in the following aspects : 
o interactive initiation into normal operation for new system operators

○ upgrade of operators' knowledge to face more challenging situations such as : rapid increase or decrease of load demand, peak load period, return to normal conditions following a contingency, etc.

o optimization of system operation: for instance, minimization of switching commands, reduction of active power losses, etc.

o training in abnormal conditions including some aspects of system restoration.

\section{THE SIMULATOR WITHIN THE CONTROL CENTER AND STUDIES ENVIRONMENTS}

Figure 3 sketches the relationship between the TS and other tools available for real-time control and planning studies. In planning and operational planning studies, extensive time simulations are performed to determine security limits with respect to angle and voltage instability and set up appropriate strategies to be used in real-time operation.

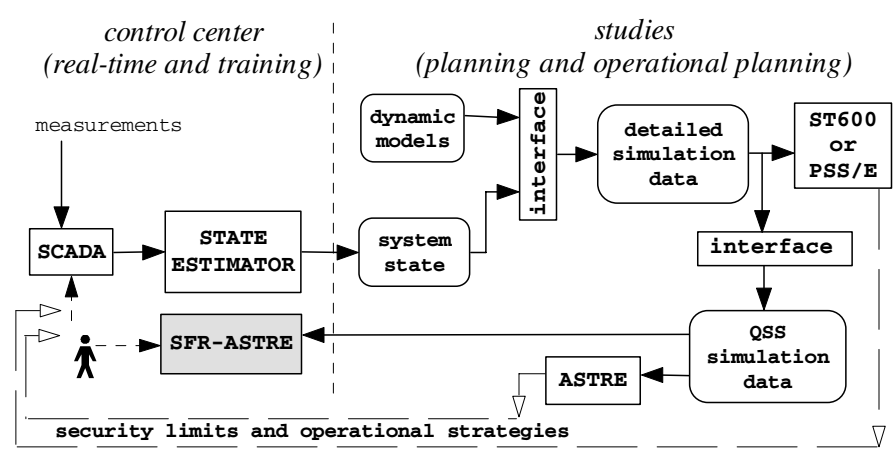

Fig.3. The training simulator in the control center and studies environments

Among them, the QSS simulation software ASTRE is routinely used in long-term voltage stability studies $[9,10]$. This tool only requires a subset of the dynamic data needed by detailed time simulation tools and an interface has been developed to automatically and transparently extract the relevant QSS data.

The development of the training simulation software from ASTRE presented several significant advantages.

First, since SFR-ASTRE uses the same data as ASTRE, direct access is given to the well validated models and data of the studies environment. The latter are readily available without adding interfaces to a specific TS format. In particular, the TS inherits the models of the system protection schemes present in the H-Q network. This includes the automatic shunt reactor tripping devices (named MAIS) that act at the $735-\mathrm{kV}$ level [13], and, in voltage instability conditions, the undervoltage load shedding scheme (referred to as TDST) [14]. The user can test the operation of these devices in virtual experiments that would not be allowed on the real system. The generation rejection and remote load shedding scheme (named RPTC) [15] can be also considered, although the short-term transients that lead to this corrective action against transient (angle) instability are not simulated. In fact, the operator must be trained to react to the consequences of RPTC.

Second, it contributes to an improved communication between the studies and real-time operation teams. The relevance of an operational guideline can be easily shown through a training session. The specific voltage stability studies and conditions that led to establishing such guidelines can be shown to the operator in order to increase his understanding of phenomena and his awareness of their importance.

Thirdly, the TS benefits from the availability of real-time snapshots of the system, through the existing link between the control center state estimator and the studies environment (see Fig. 3). Each five minutes, year long, a snapshot of the real system conditions is taken, a load flow data file is built and the corresponding dynamic data are assembled for this particular system state [16]. Using the state estimator outputs allows to present realistic training cases to the dispatchers.

Finally, the portability between the studies and the training software allows to keep the maintenance effort very low. For instance, model updates can be automatically reflected in the TS. This also reduces, by a large amount, the probability of introducing errors.

All these aspects increase the quality of the simulation, reduce the work load of preparing a training session and allow to concentrate efforts on creating new scenarios.

\section{DESIGN OF THE TRAINING SIMULATOR}

\section{A. The computational engine}

The QSS approximation relies on time-scale decomposition. Faster phenomena are represented by their equilibrium conditions instead of their full dynamics. This yields a high computational efficiency and also reduces the amount of information required in addition to network data. This method has been validated with respect to detailed time simulation for long-term voltage stability studies [9], which are typically in the same time frame as the TS.

The QSS model can be formally written as:

$$
\begin{aligned}
\boldsymbol{0} & =\boldsymbol{g}\left(\boldsymbol{x}, \boldsymbol{y}, \boldsymbol{z}_{\mathrm{c}}, \boldsymbol{z}_{d}\right) \\
\boldsymbol{0} & =\boldsymbol{f}\left(\boldsymbol{x}, \boldsymbol{y}, \boldsymbol{z}_{c}, \boldsymbol{z}_{d}\right) \\
\boldsymbol{z}_{d}(k+1) & =\boldsymbol{h}_{d}\left(\boldsymbol{x}, \boldsymbol{y}, \boldsymbol{z}_{c}, \boldsymbol{z}_{d}(k)\right) \\
\boldsymbol{z}_{c}{ }_{c} & =\boldsymbol{h}_{c}\left(\boldsymbol{x}, \boldsymbol{y}, \boldsymbol{z}_{c}, \boldsymbol{z}_{d}\right)
\end{aligned}
$$

Equation (1) stands for the network relationships and (2) for the short-term dynamics at equilibrium. Under the QSS approximation, the short-term dynamics of a generator are replaced by three nonlinear algebraic equations (2), accounting for the generation saturation, the automatic voltage regulator static gain and the governor speed droop [10]. Loads are represented as voltage dependent active and reactive powers at the $\mathrm{MV}$ buses behind the distribution 
transformers.

QSS simulation reproduces the long-term dynamics of load tap changers, overexcitation limiters, automatically switched shunt compensation, secondary voltage control (if any), protecting devices, etc. The corresponding discrete dynamics, captured by (3), involve the delays before each transformer tap change, before a synchronous machine is switched under constant field current, etc. The continuous long-term dynamics (4) stem from regulator PI control laws, inversetime characteristics, etc. In the Hydro-Québec system, a time step size of 1 second is used.

At each time step, the discrete transitions (3) are checked and applied. The new values of $\boldsymbol{x}$ and $\boldsymbol{y}$ are computed together, solving $(1,2)$ with a Newton method. A simple partitioned integration technique is used to deal with (4).

QSS simulation is much faster than real-time (simulating say - 30 minutes of the system evolution takes a few seconds). Thus, a key parameter of the TS is the ratio:

$r=($ simulated time interval) $/($ duration of simulation $)$

which can be set to 1 to render the real system evolution or to a larger value for accelerated simulation. The specified ratio is obtained by keeping the computational engine idle for an appropriate amount of time, once all the computations of the current time step are completed.

When launched, the computational engine receives the initial scenario (usually prepared by an instructor). At each time step of the simulation, it looks for commands entered by the user and, if some are found, Eqs. $(1,2)$ are solved accordingly. The discrete (3) and the continuous dynamics (4) are then taken into account. Finally, the whole system state is computed.

The QSS system evolution (more precisely, the successive values of $\boldsymbol{x}$ and $\boldsymbol{y}$ ) is stored in the computer RAM. This makes it easy to interrupt a simulation and return to a previous time or before a previous action. When the simulation is completed or interrupted, the user can inspect the time evolution of various outputs, in addition to those he selected for display during the simulation itself.

Moreover, a session with all the actions taken by the user (and the resulting events) can be stored as the initial scenario of a future session. This allows, in particular, to "replay" a former TS session, either in real time $(r=1)$ or in accelerated mode $(r>1)$.

Finally, the last steady state reached can be saved as new initial conditions, which facilitates the setting up of new scenarios.

\section{B. The server and the clients}

The TS architecture is sketched in Fig. 4. One PC hosts the computational engine, while one or several PCs connected through a Local Area Network (LAN) serve as interfaces with one or several simultaneous users. Note that the whole application can also run on a single PC, which can be a laptop.

At each time step, the computational engine dumps the whole system state in a shared memory. On the same machine, a server takes at regular intervals, a copy of this memory and distributes the relevant outputs to one or several clients.

Each client is an instance of a graphic application displaying the outputs. Each PC of the LAN can run one or several clients. The latter communicate with the server through the TCP/IP protocol. When launched, each client is "registered" by the server to whom it passes the list of outputs to be displayed. The frequency of display update is dictated by the server and corresponds to that of the SCADA system.

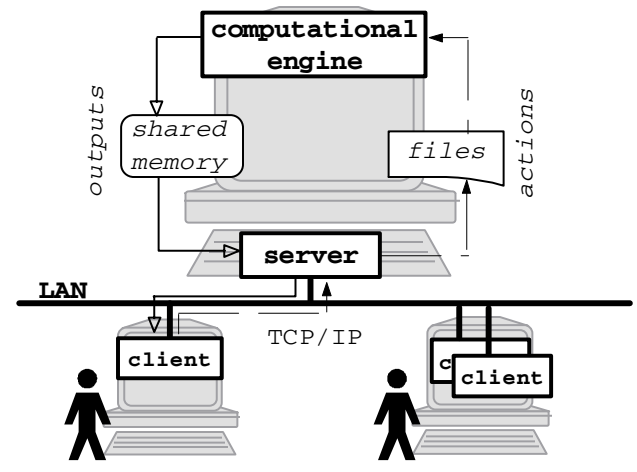

Fig. 4. Overall architecture of the training simulator

Each client also collects the actions entered by its user, writes them on a small ASCII file, and sends the latter to the server. The files so collected by the server are presented to the computational engine. From each client, actions can be sent one-by-one or grouped. A random delay of implementation can be applied to each action (corresponding to transmission delays). The lower and upper bounds of this random variable can be specified for each component of the system. A random delay, with the same bounds for all actions, can be also applied by the computational engine.

All clients have "equal rights". Hence, for instance, one client may be used by the instructor on one PC to enter disturbances, while another client is used by the trainee to react to these events. All actions are cumulated.

A new instance of the client can be launched at any time during the session, on any PC of the LAN. The various clients are served sequentially with safeguards against congestion when a client does not respond quickly enough.

The above design allows the trainee to use more displays when a more detailed training environment is sought.

\section{The Graphic User Interface (GUI)}

A simple but flexible solution has been adopted for the GUI. Each display is made up of a static background picture (in BMP or JPEG format) provided with "control points", output values, and links to other displays. An example is given by Fig. 5 which shows a fragment of the H-Q transmission system with its voltage control devices.

The small squares correspond to control points. They are coloured in green or red when they refer to power system 
equipments that can be switched on or off, and in cyan otherwise. Clicking on a control point opens a dialog window specific to the type of equipment involved. For instance, for a line, the two ending breakers can be manoeuvred, while for a group of generators, all voltages can be collectively modified by the same amount.

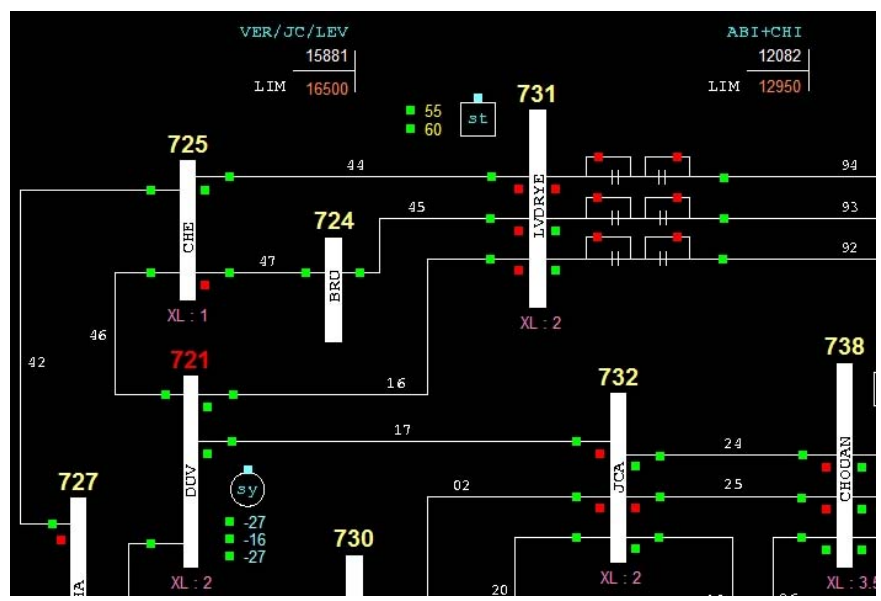

Fig.5. Example of TS display : fragment of one-line diagram.

The background picture can be produced by a variety of graphic tools. As an alternative, snapshots of the real SCADA displays can be used to mimic the control center environment. However, a display can be as simple as a table of numbers, each updated during the simulation.

The control points, output value displays and links to other displays are placed on the background picture with the help of a simple dedicated editor. When placing a control point, the designer enters the type and the name of the equipment. When placing an output value display, he specifies the type of output, the involved component as well as the colour assigned to each interval of values.

\section{A TYPICAL TRAINING SESSION}

Despite its relative simplicity, the TS supports and facilitates the different steps that lead to an efficient training session. These steps are outlined hereafter, using as an illustration the winter 2003-04 peak scenario set up some time before writing this paper.

\section{A. Identification of training objectives}

Even if obvious at first glance, the first, crucial step is to identify the training objectives. This choice must be realistic and compatible with the simulation capabilities of the tool. Different training tools are available and the appropriate one must be chosen in relation to the objectives. In the above cited example, the session aimed at preparing operators to face a period of very high load with potential difficulties to maintain adequate transmission voltages. A stand-alone TS such as SFR-ASTRE meets this objective perfectly.

\section{B. Choice of base cases and construction of scenarios}

To stay close to real operating conditions the starting point was taken from a previous peak load condition. The case was easily retrieved from the state estimator archives, according to the procedure outlined in Fig. 3. Starting from this situation, some data adjustments were performed to account for new equipments put in service since the occurrence of the reference peak load (a 735-kV transmission line, some shunt capacitors, etc.).

On top of this base case, the demand ramp, the generation adjustments and some other dynamic evolutions were specified in data files. A simulated interval of 30 to 60 minutes meets the training objectives while still allowing the storage of the whole system trajectory in the RAM, as explained in Section IV.A. When preparing the scenario, the instructor can easily adjust the system evolution to meet his training objectives, using the TS itself to observe the impact of his scenario modifications. He can perform some switching operations and save the corresponding modifications as a new base case. He can make the simulation proceed or return to a past time. He can also accelerate the speed of the simulation to have a faster overview of the case.

\section{Preparation of the training environment}

The scenarios being established, the instructor defines the displays to be presented to the trainee, as well as the setting of the training room. SFR-ASTRE easily accommodates itself to various settings, ranging from a single laptop to a very realistic environment including the projection of a map board and several specific windows. For instance, in the Winter 2003-04 peak scenario, real-time-like displays were provided with individual shunt reactor and capacitor (on/off) status together with curves showing the time evolution of the total demand and the generation of the main power plans.

\section{Training session}

Finally, the training session itself can take place. In the simplest case, the dispatcher himself may initiate the scenarios, perform switching operations through the userfriendly pop-up menus, and self-evaluate the effect of his decisions. Even in this simpler context, the TS allows him to feel the effect of the delays affecting the implementation of his commands. In more involved sessions, however, the instructor participates, either by implementing through his own displays the commands verbally transmitted by the trainee, or by applying disturbances to the system.

Once the simulation exercise is completed, the instructor can replay the simulation, show all the actions taken and discuss their efficiency. He can show on-line many aspects of the problem and its solutions. The trainee can come back to any specific time of the simulated case, try a different set of actions, compare their effects and thereby improve his skills and knowledge. 


\section{CONCLUSION}

In many power systems, voltage control has become one of the most important tasks the operator has to fulfill. A tool of the type described in this paper can significantly improve the dispatcher's ability to perform this task in an optimal manner.

The determining factor in the decision to develop this TS was the availability of a well-known study tool (subsequently transformed to render the real-time or accelerated evolution) but also the opportunity to build up a small but efficient team of persons with simultaneous expertise in control centers, power system dynamics and computers. The confidence into the ability to deliver the TS rapidly and with reasonable efforts was gained from the previous success of this university-company collaboration.

High flexibility was achieved with low software development efforts. Although "light", the resulting application is realistic. Its design makes it easy to use, and facilitates the development of specific training sessions, while relying on standard computer hardware.

Requirements specific to the Hydro-Québec were also met, regarding for instance system protection schemes.

As for any TS, an important feature is the ability to retrieve real system states from the state estimator archives, so that real-life network conditions are considered in the training sessions. Also, a typical training session must be well prepared and organized in terms of training objectives, base case and scenarios.

While the focus of the paper was to show that this TS adequately meets to Hydro-Québec voltage control needs, the use of this software is not limited to this objective nor to this system. For instance, the simulation of the long-term dynamics of frequency is presently being envisaged.

\section{ACKNOWLEDGMENTS}

The authors gratefully acknowledge the involvement of Lorraine Marsolais, Jacques Gauthier and Laval Riverin in this project. François Lévesque is heartily thanked for providing human resources.

\section{REFERENCES}

[1] C. Jeanbart, Y. Logeay, M. Musart, «EDF simulator for control center operators », CIGRE report CE 39-12, 1988

[2] G.W. Cauley, C.J. Frank, D.H. Curtice, "Operator training simulator", EPRI Final Report EL-7244, Vol. 1, 1991

[3] N. Wang, R. Cheung, G. Wu, J. Naccarino, J. Castle, "Simulation of the New York power pool for dispatcher training", IEEE Trans. on Power Systems, Vol. 9, No. 4, pp. 2063-2072, 1994

[4] S.V. Vadari, M.J. Montstream, H.B. Ross, "An online dispatcher training simulator function for real-time analysis and training", IEEE Trans. on Power Systems, Vol. 10, No. 4, pp. 1798-1804, 1995

[5] P. Kambale, J.J. Mackaer, "The dispatcher training simulator for Metropolitan Edison Company", IEEE Trans. Power Systems, Vol. 11, pp. 898-904, 1996

[6] D.L. Fletcher, J.G. Coleman, "The dispatcher training simulator at PECO Energy company", IEEE Trans. on Power Systems, Vol. 13, No. 3, pp. $1150-1155,1998$
[7] K. Walve, A. Edström, "The training simulator ARISTO - design and experience", Proc.1999 IEEE PES winter meeting, pp. 545-547

[8] S. Gissinger, P. Chaumès, J.-P. Antoine, A. Bihain, M. Stubbe "Advanced dispatcher training simulator", IEEE Computer Applications in Power, Vol. 13, No. 2, pp. 25-30, 2000

[9] T. Van Cutsem, R. Mailhot, "Validation of a fast voltage stability analysis method on the Hydro-Québec system," IEEE Trans. Power Systems, Vol.12, pp. 282-292, 1997

[10] T. Van Cutsem, C. Vournas, Voltage Stability of Electric Power Systems, Kluwer Academic Publishers, Boston, 1998

[11] J-P. Gingras, R. Mailhot, and J. Lavigne, "Review of the Hydro-Québec system operation with series compensation", CIGRE report 38-101, 2000

[12] S. Guillon, S.Harrison, A. Lapointe, F. Lévesque, "Implementation of indices in the control center, in order to prevent a degrading voltage robustness of the power network during load variations," Proc. of the CIGRE-IEEE Int. Symp. on Quality and Security of electric power delivery systems, Montréal, Oct 2003, pp. 2-206

[13] S. Bernard, G. Trudel, and G. Scott, "A 735 kV Shunt Reactors Automatic Switching system for Hydro-Québec Network", IEEE Trans. on Power Systems, Vol.11, pp. 2024-2030, 1996

[14] D. Lefebvre, S. Bernard, T. Van Cutsem, "Undervoltage load shedding scheme for the Hydro-Québec system", paper to be presented at the panel session on undervoltage load shedding, IEEE PES general meeting, Denver, June 2004

[15] S. Bernard, G. Trudel, and G. Scott, "Hydro-Québec's Defense Plan Against Extreme Contingencies," IEEE Trans. on Power Systems, Vol. 14, No 3, pp. 958-965, 1999

[16] L. Riverin, A. Valette, "Automation of security assessment for HydroQuébec's power system in short-term and real-time modes", CIGRE report 39-103, 1998

Thierry Van Cutsem (M'94, SM'03) graduated in Electrical-Mechanical Engineering from the University of Liège in 1979, where he obtained the Ph.D. degree in 1984 and is now adjunct professor. Since 1980 he has been with the Belgian National Fund for Scientific Research (FNRS), of which he is now a Research Director. His research interests are in power system dynamics, stability, security and real-time control, in particular voltage stability and security

Gaëtan Hassé graduated in Electrical-Mechanical engineering from the University of Liège in 2000, where he has been working as a research engineer from 2000 to 2003 on the OMASES and SFR-ASTRE projects. He is now with the Tractebel company.

Cédric Moors received the Electrical Engineering diploma (power systems orientation) in 1998 and the Ph.D. degree in 2002, from the University of Liège. His main research area has been the emergency control of voltage instability. Since 2003, he is with ELIA, the Belgian TSO.

Sébastien Guillon (M'2003, GOLD member) graduated from Ecole Polytechnique de Montréal, Canada, in Physics Engineering and has a master in Theoretical Physics from Concordia University. His experience is mainly with the planning department of the TransÉnergie division of Hydro-Québec. His interests are in voltage stability, operational planning practices and control systems.

Richard Mailhot graduated in Electrical Engineering from Sherbrooke University, Canada, in 1981. He has many years of experience in system planning and operation. He presently leads the Support and Integration group of Hydro-Québec control center. 